\title{
Interfaces Cerebro-Computadora: Conceptualización, Retos de Rediseño e Impacto Social
}

\section{eBrain Computer-Interfaces: Conceptualization, Redesign Challenges and Social Impact}

\author{
L. M. Alonso-Valerdi', M. A. Arreola-Villarruel², J. Argüello-García \\ 'Tecnológico de Monterrey \\ ¿Universidad de Guadalajara \\ ${ }^{3}$ Instituto Politécnico Nacional
}

\section{RESUMEN}

Las Interfaces Cerebro-Computadora (ICCs) son sistemas que miden la actividad del Sistema Nervioso Central y la convierten en salidas que reemplazan, restauran, aumentan, suplementan o mejoran las salidas naturales de dicho sistema y, por lo tanto, cambian las interacciones en curso entre el ser humano y su ambiente interno o externo. Sin embargo, a más de medio siglo de ser investigadas en condiciones de laboratorio, las ICC no han podido ser trasladadas al mundo real. En el presente artículo de revisión se busca: (1) comprender a fondo la estructura de una ICC y los tipos de sistema, (2) analizar el reto que tiene la comunidad científica para mejorar la interacción entre usuario y sistema desde la perspectiva de la ingeniería de factores humanos y (3) describir la aplicación de este tipo de tecnología de asistencia en desarrollo en la sociedad mexicana. El futuro de estas ICC y la eficiencia con que logren el objetivo para el que fueron diseñadas, parece depender más que nunca de factores relacionados a la percepción subjetiva del usuario, su adaptación al manejo de las ICC y el proceso de internalizarlas como propias en su espacio personal y su psique.

PALABRAS CLAVE: Interfaz Cerebro-Computadora; Mapeo Transparente; Sentido de Autonomía; Retroalimentación Multisensorial; Ambiente Ecológico 


\section{ABSTRACT}

Brain-Computer Interfaces (BCIs) record the neural activity of the Central Nervous System, and then, produce outputs that replace, restore, increase, supplement or improve the natural outputs of such system. Therefore, the interaction between the human beings and their internal or external environment is tranformed. However, over a century being investigated under laboratory conditions, BCIs have not been able to be transferred to the real world. This review aims to: (1) thoroughly understand the structure of a BCI and the system types, (2) analyze the challenge that the scientific community is facing to improve the interaction between user and system from the perspective of the engineering of human factors; and (3) describe the application of this type of assistive technology under development in the Mexican Society. The future of this technology and its effectiveness seem to depend more than ever on factors related to the user subjective perception, and the user adaptation to the system.

KEYwORDS: Brain Computer-Interface; Transparent Mapping; Sense of Agency; Multisensorial Feedback; Ecological Environment

\section{Correspondencia}

DESTINATARIO: Luz María Alonso Valerdi

INSTITUCIÓN: ecnológico de Monterrey

DIRECCIÓN: Av. Eugenio Garza Sada \#2501 Sur, Col.

Tecnológico, C. P. 64849, Monterrey, Nuevo León

CORREO ELECTRÓNICO: Im.aloval@tec.mx

\section{Fecha de recepción:}

15 de junio de 2019

Fecha de aceptación:

15 de agosto de 2019 


\section{INTRODUCCIÓN}

Una Interfaz Cerebro-Computadora (ICC) es un sistema que decodifica la actividad neuronal del usuario (ej., señales eléctricas, campos magnéticos, flujo sanguíneo), con la finalidad de controlar dispositivos (principalmente prótesis o sillas de ruedas) que permitan devolverle la independencia a personas que han perdido la capacidad de auto cuidarse ${ }^{[1]}$. El interés en la implementación de sistemas que pudiesen establecer comunicación directa con el cerebro humano ha crecido a tal magnitud que a la fecha se han propuesto aplicaciones para el diagnóstico, tratamiento y seguimiento de una gran variedad de enfermedades con afectación neurofisiológica y neuropsicológica [2]. Algunas propuestas relevantes han sido la detección de niveles de conciencia en pacientes en coma, estado vegetativo o estado de conciencia mínima ${ }^{[3]}$; el incremento de los niveles de atención en niños con desórdenes de hiperactividad y déficits de atención [4]; la regulación cognitiva y afectiva de personas con esquizofrenia o epilepsia [5]; y la mejora de la interacción social de niños con autismo ${ }^{[6]}$. El interés de la comunidad científica por las ICC también se ve reflejado en la cantidad de artículos publicados y número de grupos de investigación trabajando en ellas. Del mismo modo, la creación de nuevos grupos de investigación es un indicador adicional de la atención recibida. En el primer congreso internacional sobre ICC, llevado a cabo en 1999 en los Estados Unidos, se presentaron alrededor de 50 participantes de 22 grupos de investigación, mientras que en la edición del 2016 asistieron 400 participantes de 188 grupos de investigación y otras organizaciones $\left.{ }^{[7]}\right)$. Para ${ }^{[8]}$, la atención que han recibido las ICC puede ser atribuida a la coincidencia de los siguientes factores: computadoras más rápidas y económicas, avances en nuestra comprensión y conocimiento de cómo nuestro cerebro procesa la información sensorial y produce respuestas motoras, mayor disponibilidad de dispositivos para registrar señales cerebrales, procesamiento de señales más poderoso y algoritmos de aprendizaje automático más eficientes y eficaces.
La investigación en sistemas ICC comenzó aproximadamente en los años setentas ${ }^{[1]}$. Sin embargo, todavía distan no solo de ser una tecnología de asistencia real, sino incluso de ser un prototipo complemente funcional para cualquier tipo de persona lo que requiriese este tipo de tecnología. A pesar de que se ha avanzado enormemente en el desarrollo tecnológico (ej., sensores, sistemas de adquisición, modelados matemáticos, algoritmos computacionales), aún no se logra decodificar con precisión la información encriptada en la actividad neuronal del cerebro humano. A partir del 2010, en la comunidad científica dedicada a la investigación en sistemas ICC se ha acuñado el término "analfabetismo ICC" para denominar a toda persona que es entrenada para controlar un sistema ICC, pero que aún después de una capacitación exhaustiva no logra establecer comunicación con él. De forma global, entre el $15 \%$ y el $30 \%$ de los usuarios no logran controlar un sistema ICC, mientras que el resto (aproximadamente el 80\%) alcanzan un desempeño moderado ${ }^{[9]}$. Algunos investigadores se han dedicado a averiguar el motivo de estos altos índices de deficiencia de los sistemas ICC y han sugerido las causas discutidas a continuación.

\section{Variabilidad de las Señales de EEG}

Una de las formas más típicas de detectar la actividad neuronal es a través del registro de señales de electroencefalografía (EEG), ya que es un método relativamente económico, versátil y no invasivo. En los últimos años, se ha demostrado que las ICC que detectan la actividad neuronal a través de EEG no son sistemas estándares, por el contrario, cada módulo debe ser ajustado a cada usuario. Debido a la gran variabilidad de las señales de EEG, el funcionamiento de una ICC no es reproducible entre usuarios y, en muchas ocasiones, ni siquiera con un mismo usuario.

\section{Insuficiencia de Información}

La naturaleza de las señales de EEG (no estacionarias, variantes en el tiempo, no lineales, aleatorias, entre otras características) ha complicado la extracción de 
información suficiente que permita determinar con precisión las intenciones del usuario al momento de establecer comunicación con el sistema. En este aspecto, un intento por mejorar la decodificación de las intenciones del usuario ha sido la inclusión de otras señales fisiológicas como la actividad eléctrica ocular, muscular y del corazón, además del ritmo respiratorio, la conductancia de la piel y la presión cardiovascular ${ }^{[10]}$. Este tipo de sistemas se conocen como sistemas ICC híbridos.

\section{Interacción entre el Usuario y el Sistema}

A la fecha, se ha comprobado que el protocolo tradicional de entrenamiento que se usa para lograr que una persona llegue a modular su actividad neuronal con el objetivo de posteriormente controlar un sistema ICC es poco pedagógico, incluso para propósitos distintos a los establecidos en la investigación de sistemas ICC ${ }^{[11]}$. Se ha señalado que la principal deficiencia del proceso de entrenamiento es la nula atención que se le ha puesto al rol primordial que juega la psicología educativa, la ergonomía, el diseño interactivo y la ingeniería de factores humanos ${ }^{[12]}$. Los protocolos estandarizados simplemente no se alinean con los principios de aprendizaje humano. Algunas propuestas actuales han sugerido la incorporación de sistemas tutoriales inteligentes que den soporte personalizado durante el proceso de aprendizaje y que a la par, proporcionen un proceso de entrenamiento que se ajuste al perfil del aprendiz y a la evolución de sus habilidades [13]. Mientras los sistemas ICC no sean diseñados en base a las necesidades de los usuarios, ellos seguirán teniendo habilidades limitadas (en el mejor de los casos) y hasta el algoritmo computacional más sofisticado fallará ${ }^{[14]}$.

Las técnicas de entrenamiento de los usuarios para controlar un sistema ICC ha sido escasamente estudiada en la literatura, y la mejor forma de entrenar a los usuarios para desarrollar sus habilidades de control sigue siendo desconocida. Algunos de los avances en este tema han sido; por un lado, la propuesta de dife- rentes métodos para clasificar a los individuos como usuarios de alta o baja aptitud y de este modo evitar el consumo de tiempo adaptando a un usuario que probablemente requiera otro tipo de entrenamiento y/o sistema. Por otro lado, se ha mencionado en recientes investigaciones que el desempeño de un sistema ICC puede mejorar, con el simple hecho de proporcionar al usuario instrucciones específicas sobre las tareas de control a desempeñar ${ }^{[15]}$.

En base a lo anteriormente expuesto, en el presente artículo de revisión se busca: (1) comprender a fondo la estructura de una ICC y los tipos de sistema (Conceptualización), (2) analizar el reto que tiene la comunidad científica para mejorar la interacción entre usuario y sistema desde la perspectiva de la ingeniería de factores humanos (Retos de Rediseño) y (3) describir la aplicación de este tipo de tecnología de asistencia en desarrollo en la sociedad mexicana (Impacto Social). Con respecto al segundo punto, los factores humanos que se analizarán son: (1) mapeo transparente, (2) sentido de autonomía, (3) retroalimentación multisensorial y (4) ambientes ecológicos. Por último y para concluir con el presente trabajo, se discutirán los puntos más relevantes de la revisión en términos de la exclusión del usuario en el diseño de las ICCs, como posible causa del bajo desempeño del sistema.

\section{CONCEPTUALIZACIÓN}

De forma más puntual, una ICC es un sistema que mide actividad del Sistema Nervioso Central (SNC) y la convierte en salidas artificiales que reemplazan, restauran, mejoran y suplementan salidas naturales por parte del SNC. Esto significa que las interacciones que ocurren entre el SNC y el ambiente externo se realizan a través de la ICC [16]. En general, se pueden describir cuatro tipos de interfaces: activas, reactivas, pasivas e híbridas [17]. En la Figura 1 se esquematizan estas cuatro clases de ICCs y se resumen sus características principales. A continuación, se presenta una breve descripción de cada tipo de sistema. 


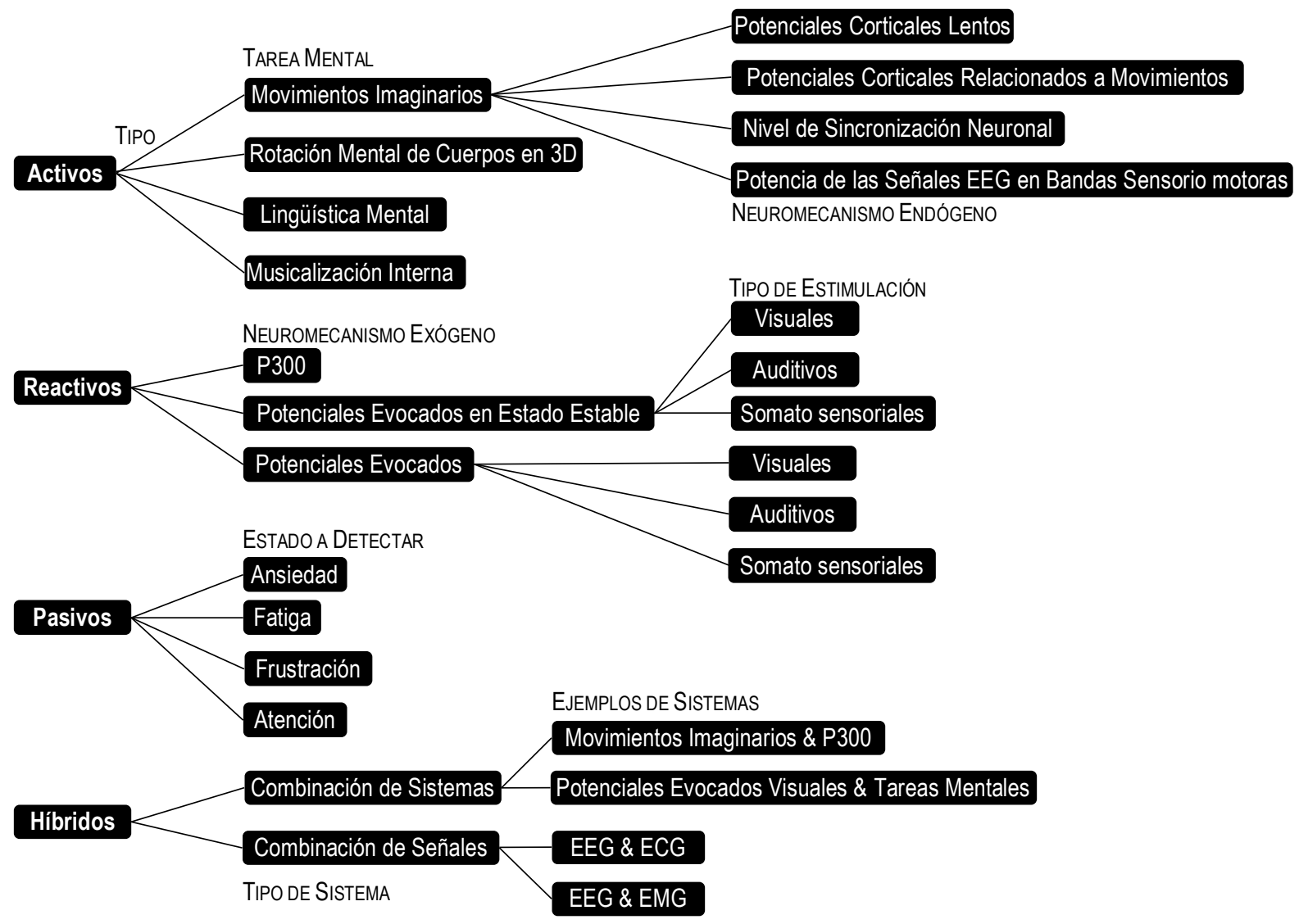

FIGURA 1. Tipos de sistemas ICCs: activos, reactivos, pasivos e híbridos. Los sistemas activos se controlan a voluntad del usuario, los reactivos dependen de un ciclo emisión-recepción, los pasivos proporcionan información sobre el estado cognitivo del usuario y los híbridos extraen información de diversas fuentes fisiológicas.

Los sistemas activos son aquellos que obtienen sus salidas mediante el control consciente por parte del usuario. La principal característica de este tipo de sistemas es la libertad que se le proporciona al usuario para manipular una aplicación o dispositivo externo.

Los sistemas reactivos se caracterizan por ofrecer aplicaciones controladas mediante la reacción del usuario ante un estímulo sensorial externo: visual, auditivo, táctil u olfativo. Aunque este tipo de sistemas se controlan externamente; es decir, se depende permanentemente de la emisión (sistema) y recepción (usuario) de estímulos externos, su ventaja principal es que no necesitan un entrenamiento previo y arduo para que el usuario produzca la respuesta neuronal deseada.
Los sistemas pasivos no controlan directamente un dispositivo objetivo. La adquisición y análisis de la actividad neuronal se realiza, básicamente, para descifrar un estado cognitivo del sujeto (ej., cansancio, sueño, nivel de atención, etc.). Los datos sirven para modificar el control del dispositivo objetivo, el cual no depende de la actividad neuronal obtenida. Estos sistemas han sido muy explotados para enriquecer la interacción humano-computadora.

Por último, los sistemas híbridos se caracterizan por fusionar mecanismos neurofisiológicos de ICCs activas y reactivas para mejorar su desempeño. También se refieren a sistemas que usan otros tipos de señales fisiológicas, como ritmo cardiaco, respiración o con- 
ductancia de la piel, en conjunto con la actividad neuronal para, también, incrementar su desempeño. Nótese que estos sistemas sí controlan directamente el dispositivo o aplicación objetivo, solo que lo hacen a través de diversas fuentes fisiológicas. Independientemente del tipo de sistema, todas las ICCs se conforman, esencialmente, de diez módulos: usuario, detec- tor de actividad neuronal (EEG para sistemas no invasivos), procesador de señales, generador de características, traductor de características, sistema de control, retroalimentación, controladores del dispositivo, dispositivo o aplicación a controlar y monitor neuronal, aunque éste último no es tan esencial como todos los anteriores (refiérase a la Figura 2).

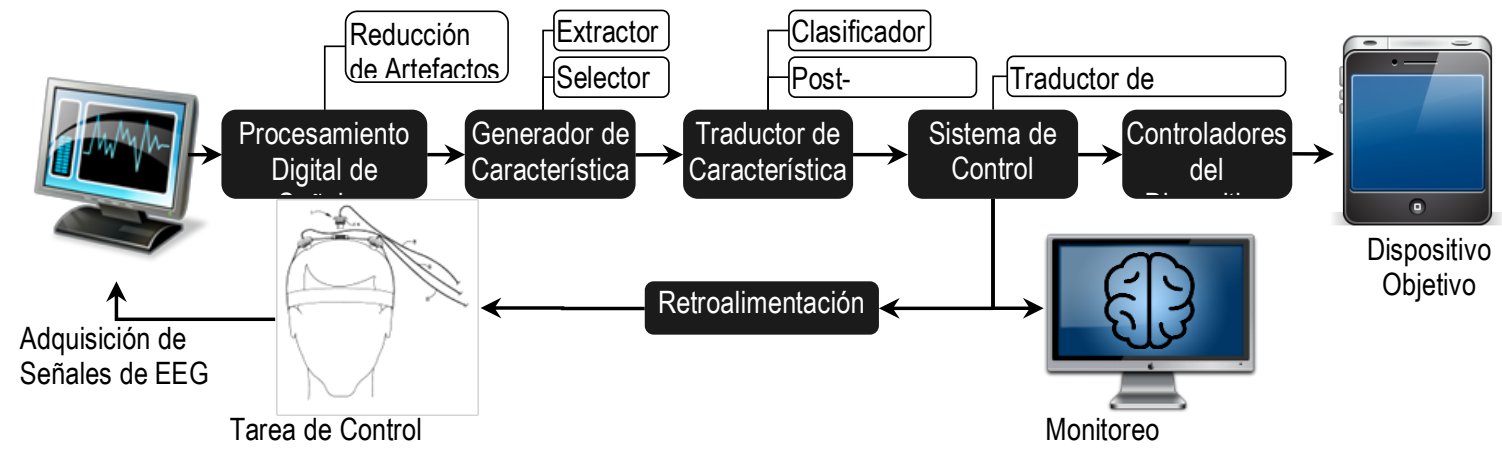

FIGURA 2. Esquema general de un sistema ICC. En general, estos sistemas se componen del (1) usuario,

(2) EEG, (3) procesador digital de señales, (4) generador de características, (5) traductor de características, (6) sistema de control, (7) retroalimentación, (8) controladores, (9) dispositivo o aplicación objetivo y (10) monitor neuronal.

Un sistema ICC convierte las intenciones de un usuario (tarea de control) codificados en un neuromecanismo específico (véase Figura 1) en acciones reales en seis fases fundamentales. Primero, se adquiere la actividad cerebral que se logra a través de diversas técnicas de registro que se agrupan en tres categorías en función del tipo de actividad cerebral que capturan: eléctrica, química o metabólica. Cada una de las técnicas tiene características que las hacen idóneas según la información que deseemos obtener. Por ejemplo, la EEG registra la actividad eléctrica cerebral por medio de electrodos colocados en la superficie del cuero cabelludo y representa la suma de los potenciales post-sinápticos de las neuronas piramidales, principalmente, de la corteza cerebral. La EEG es considerada como la única técnica capaz de ser implementada hoy en día en un sistema ICC en tiempo real fuera de laboratorio, debido a que es una técnica no-invasiva, con alta resolución temporal y existen equipos portátiles de bajo costo. Una vez seleccionada la técnica de registro, el siguiente paso consiste en procesar la información adquirida con el objetivo principal de eliminar artefactos tanto internos (ej., otras señales electrofisiológicas principalmente la ocular, la miográfica y la cardíaca) como externos (ej., campos electromagnéticos a $60 \mathrm{~Hz}$ ). El tercer paso, consiste en extraer y seleccionar características. De éstas existen dos tipos: simples y complejas. Las características simples son una medición directa de la señal (ej., amplitud), mientras que las complejas son combinaciones lineales o no lineales, razones, estadísticos u otras transformaciones de las características simples. Si se seleccionan adecuadamente, las características complejas pueden reflejar las intenciones del usuario con mayor precisión que las simples. Aun así, la mayoría de los sistemas ICC extrae un conjunto de características simultáneamente. Después de haber identificado y estimado las características eléctricas que describen la actividad neuronal a estudiar, se reconocen patrones neuronales con la ayuda de algoritmos de aprendizaje automático. 
El campo del reconocimiento de patrones se relaciona con el descubrimiento automático de regularidades en los datos a través del uso de algoritmos computacionales, y con el uso de esas regularidades para realizar acciones, como la clasificación de datos en distintas categorías ${ }^{[18]}$. En un sistema ICC, las categorías corresponden a los estados sensoriales, motores, cognitivos o emocionales que se desean identificar en el usuario del sistema ICC. Posteriormente, en función de la clase detectada, se ejecuta una acción predefinida. Por último, en la etapa de retroalimentación, se presenta información al usuario para notificarle sobre el efecto que se desencadenó como resultado de su estado mental (sensorial, motor, emocional o cognitivo) identificado por el sistema ICC.

El presente trabajo focaliza su interés en los sistemas activos por dos razones primordiales: neuroplasticidad y autonomía. Por un lado, las tareas de control, principalmente aquellas que se asocian con imaginación motora (IM), modifican la neuroplasticidad y dicho efecto adyacente puede ser aprovechado para otras aplicaciones de los sistemas ICC como en la Neurorehabilitación. Por otro lado, los sistemas activos son los únicos que pueden llegar a proporcionar autonomía a los usuarios a la hora de intentar controlar al dispositivo objetivo. Por consiguiente, se resumen a continuación las características principales de las ICC activas controladas por IM.

\section{Sistemas ICC activos controlados por IM}

Para controlar una ICC, es necesario que el usuario genere patrones neuronales distintivos que caractericen, y diferencien sus estados mentales a identificar. Con este propósito se eligen uno de dos tipos de tareas de control: exógenas o endógenas. Las tareas de control exógenas canalizan la atención del usuario por medio de estímulos visuales, auditivos y/o táctiles (ICC reactivas), mientras que las endógenas son refieren a esfuerzos mentales que modulan la actividad neuronal significantemente (ICC activas). A lo largo de los años distintas tareas han sido propuestas y probadas, siendo la IM una de las más utilizadas en términos de las tareas de control endógenas. La IM es el ensayo mental de actos motores simples o complejos que no van acompañados de movimientos corporales manifiestos ${ }^{[19]}$. La IM modifica la actividad neuronal en las áreas sensoriomotoras primarias de una manera muy similar a la observada durante la ejecución de un movimiento real [20] [21]. Debido a esta capacidad de generar patrones reconocibles en función del movimiento imaginario, ha sido utilizada ampliamente como tarea de control en ICC [22] [23] [24]. Para [20], la IM implica sentirse ejecutando una determinada acción, ya sea que involucre todo el cuerpo o solo una parte de este. Además, es posible hacer una distinción entre dos clases: la imaginería interna y la imaginería externa. La primera, requiere imaginar el movimiento y las sensaciones físicas y emocionales experimentadas durante la acción desde una óptica en primera persona. La segunda, sin embargo, requiere imaginar un movimiento desde la perspectiva en tercera persona ${ }^{[25]}$. A diferencia de otros tipos de imaginación, como la visual, la IM es difícil de describir verbalmente. Empero, es generalmente caracterizada por representaciones mentales vívidas y por cambios en el ritmo respiratorio y cardíaco que están relacionados con el grado de esfuerzo mental ${ }^{[19]}$. Estudios como los de ${ }^{[26]}$ demuestran que la IM involucra secciones somatotópicamente organizadas de la corteza motora primaria de una manera sistemática y, además, regiones específicas a partes corporales en las áreas motoras no primarias. Estos hallazgos muestran que la IM de diferentes tipos de movimiento activa las representaciones motoras correspondientes ${ }^{[27][28][29]}$.

La detección de movimientos imaginarios de un individuo es parte de una tarea retórica, tanto que, incluso, se ha convertido en el tema fundamental de algunas investigaciones ${ }^{[5]}$. El estudio de las señales de EEG modulada por actividad motora se realiza esencialmente en función de dos patrones: niveles de sincroni- 
zación y desincronización neuronales (ERD/ERS, por sus siglas en inglés: Event-Related Desynchronization y Event-Related Synchronization, respectivamente) y potenciales corticales relacionados a movimiento (MRCP, por sus siglas en inglés: Movement-Related Cortical Potentials). Ambos patrones difieren entre sí en las características y las causas que los originan ${ }^{[30]}$.

\section{Sincronización y desincronización relacionadas a eventos (ERD/ERS)}

Un ERD/ERS representa un cambio específico en la frecuencia actual de la actividad EEG y puede mostrar un incremento o decremento de la potencia de una banda de frecuencia específica. Debido a que la amplitud de la señal cortical es proporcional al número de las neuronas activas, se cree que el incremento/decremento en la potencia del registro EEG se debe a la sincronización/desincronización de la población de neuronas. Para detectar el proceso ERD/ERS, la señal de EEG debe ser analizada en el dominio de la frecuencia, concentrándose en aquellas bandas asociadas al movimiento: $\mu$ (8-12 Hz) $\beta(16-24 \mathrm{~Hz})$ y $\gamma(\approx 40 \mathrm{~Hz})^{[31]}$.

Se ha propuesto que la desincronización previa al movimiento relacionada con los movimientos breves imaginarios y la sincronización post-movimiento pueden llegar a ser patrones que diferencian eficientemente distintos movimientos ${ }^{[32]}$. De hecho, algunos investigadores han encontrado que, usando tres canales de EEG, la mayoría de las características empleadas como discriminantes entre tres clases, incluyendo el estado "no controlado", fueron post-movimientos imaginarios, en lugar de características peri-movimientos imaginarios (es decir, durante el movimiento imaginario per se). También se ha sugerido que el entrenamiento basado en dos estados cognitivos básicos, movimiento imaginario (ERD) y relajación (ERS), mejoran en su totalidad la presencia de la onda $\mu{ }^{\text {[33]. }}$ Todavía más, de acuerdo a ${ }^{[15]}$, entre más específica sea la tarea motora imaginaria, mayor será la potencia de la onda $\mu$.

\section{Potenciales corticales relacionados a movimiento (MRCP)}

Un MRCP es un conjunto de cambios en el potencial de la actividad cortical que ocurren antes y después del inicio del movimiento y su registro se realiza en el dominio del tiempo. Los componentes más relevantes de este patrón son los Bereitschaft Potentials (BP) y los potenciales motores (MP, por sus siglas en inglés: Motor Potentials).

Los BPs usualmente se dividen en potenciales tempranos y tardíos ${ }^{[34]}$. Por un lado, los componentes tempranos del BP generalmente comienzan entre 1 y 2 s previos al inicio del movimiento, siendo bilaterales y simétricos a lo largo del cráneo, con una amplitud máxima alcanzada sobre el vértice $(\mathrm{Cz})$. Este componente refleja el proceso de preparación motora, el cual debe operar en ambas situaciones, cuando sólo se imagina y cuando existe un movimiento real (área motora suplementaria). Al comparar un evento imaginario con la realización del movimiento, no existen diferencias significativas entre las características espaciales y temporales de los componentes tempranos de los potenciales. Un efecto del aprendizaje adquirido en los componentes tempranos de los potenciales, está asociado con los movimientos imaginarios. Este componente probablemente refleja los procesos de preparación directamente relacionados con el movimiento, más que simplemente la atención o anticipación de la respuesta. Por otro lado, los componentes tardíos del BP usualmente comienzan dentro de los 500ms previos al inicio del movimiento. Con respecto al MP, este componente refleja la actividad cortical asociada con la ejecución del movimiento, proviniendo predominantemente del área motora primaria contralateral. El MP además refleja, en su mayoría, la actividad de la corteza motora primaria asociada con la ejecución del movimiento [35].

En movimientos de manos y dedos, las amplitudes máximas alcanzadas de los MRCPs son detectadas en la porción del cráneo que es contralateral a la que efec- 
túa el movimiento, mientras que, para las porciones distales de los miembros inferiores, las amplitudes máximas de los MRCPs son detectados en la porción ipsilateral. Cabe destacar que los MRCPs de movimientos imaginarios son más sensibles a la velocidad, que al nivel de fuerza ${ }^{[36]}$.

\section{Características de un sistema ICC basado en la detección de patrones motores}

Procesamiento Digital de Señales. Hasta el momento, los filtros espaciales comunes y los análisis discriminantes lineales se han considerado las mejores herramientas para la clasificación de tareas de control basadas en IM.

Retroalimentación. Se ha sugerido que la modalidad de retroalimentación usada en los sistemas ICC debe ser personalizada y que además se requieren nuevas estrategias para su selección [37]. Se ha encontrado que se requieren al menos 4 sesiones para observar un proceso de aprendizaje y, por lo tanto, para evaluar una modalidad de retroalimentación en particular. La retroalimentación es esencial para aprender a operar un sistema ICC, puesto que no es claro para el usuario saber desde un principio que es exactamente lo que tiene que hacer y como lo tenía que hacer para lograr que la computadora interprete adecuadamente sus tareas de control. En este tema, el uso de retroalimentación táctil ha arrojado resultados comparables, o aún mejores que aquellos obtenidos con retroalimentación visual. El uso de la modalidad auditiva como medio de retroalimentación parece no ser tan prometedora, pues el desempeño del sistema es menor al de la modalidad visual. Recientemente, se ha propuesto la retroalimentación multidimensional para mejorar el desempeño del sistema, pero esta es aún una hipótesis no comprobada [38] [39].

Estrategias Metodológicas. En los últimos años, se ha enfatizado que los protocolos estándares deberían ser reemplazados por ambientes interactivos, entrena- mientos motivantes y retroalimentación multimodal. Por ejemplo, algunos estudios muestran que el desempeño puede mejorar si se emplea una retroalimentación parcial; es decir, hacer creer al usuario que su desempeño fue mejor de lo que en realidad lo fue [40]. Este punto se discutirá con mayor profundidad en la siguiente sección.

\section{RETOS DE REDISEÑO}

Como se definió previamente, las ICCs son sistemas que miden la actividad del SNC y la convierten en salidas que reemplazan, restauran, aumentan, suplemen$\tan$ o mejoran las salidas naturales del SNC y, por lo tanto, cambian las interacciones en curso entre el ser humano y su ambiente interno o externo ${ }^{[41]}$. Debido a la posibilidad de las ICC de establecer un nuevo medio de comunicación, independientemente del sistema motor, la población que más podría beneficiarse con los avances en el área es aquella con déficits motores, como se discutirá en la siguiente sección. Sin embargo, a más de medio siglo de ser investigadas en condiciones de laboratorio, las ICC no han podido ser trasladadas al mundo real. Una de las razones principales es su bajo porcentaje de clasificaciones correctas en relación con la intención del usuario. En ${ }^{[42]}$, se argumenta que podría deberse a la naturaleza de las tareas de control que realizan los usuarios con el fin de controlar las ICC.

Como se mencionó anteriormente, las tareas de control se refieren a la estrategia aplicada para modular la actividad neuronal del usuario, de tal modo que logren generar patrones neurofisiológicos identificables que reflejen las intenciones de control de este. Con el objetivo de evocar patrones reconocibles, las tareas de control generalmente no conllevan una interacción natural entre el usuario y el sistema. Es decir, las tareas de control no son transparentes, son inconsistentes y no producen sentido de autonomía, entendido este como la autopercepción de que en verdad se realiza la acción. Un claro y clásico ejemplo es el panel de control con cuatro grados de libertad diseñado en base 
a IM, donde: (1) arriba se decodifica por movimientos imaginarios de la lengua, (2) abajo se asocia con movimientos de los pies, (3) izquierda con movimientos arbitrarios de la mano (o el brazo) izquierda, y (4) derecha con aquellos de la mano derecha. Como se puede constatar en este ejemplo, las tareas de control (movimientos imaginarios) y los comandos de control (direccionamientos) no son transparentes porque los movimientos imaginarios no son efectuados como tales, y son inconsistentes porque la asociación (arriba, abajo, izquierda, y derecha) es arbitraria. La carencia de intuición y naturalidad para controlar los sistemas ICC podrían resultar en un alto índice de usuarios que no logran establecer comunicación, aún después de un arduo entrenamiento ${ }^{[43] .}$

Por lo anterior, en esta sección nos proponemos discutir los avances que se han realizado sobre la optimización de ICC activas controladas por IM, considerando cuatro factores principales: (1) mapeo transparente, (2) sentido de autonomía, (3) retroalimentación multisensorial, y (4) ambientes ecológicos. Como se describirá a continuación, estos factores se asocian directamente con la entidad más importante de un sistema ICC: el usuario. A la fecha, la mayoría de las investigaciones están direccionadas a la optimización de los módulos de una ICC. Sin embargo, los factores humanos generalmente son ignorados al momento de implementar un sistema de este tipo. Por consiguiente, es de crucial importancia realizar una revisión de los términos y condiciones que se necesitan cumplir mínimamente para satisfacer los requisitos del usuario, quien determina generalmente la calidad de comunicación en una ICC.

\section{Mapeo Transparente}

El mapeo entre una tarea mental y la acción realizada es denominado "transparente" cuando la acción realizada conforma la tarea mental ${ }^{[44]}$. Por ejemplo, si imaginamos que giramos la perilla de una puerta para abrirla y, posteriormente, abrimos la puerta girando la perilla, el pensamiento y la acción se "ajustan” el uno al otro. En los sistemas ICC, esta "transparencia" entre tarea mental y comando de control es poco utilizada. Principalmente, debido a que los investigadores han preferido maximizar la eficiencia en la distinción entre tarea y comando de control, utilizando tareas mentales que generen la mayor diferencia posible en cuanto a la actividad cerebral generada por cada una, sin preocuparse demasiado por la congruencia tarea-acción. No obstante, si el sistema hace lo que el usuario quiere que haga, este tiene la sensación de tener el control o de que está controlando el sistema ${ }^{[45]}$.

Quizá el paradigma más conocido y utilizado en ICC sea el de Graz ${ }^{[46]}$, donde se le pide al participante imaginar alguna de las siguientes combinaciones de movimientos con la finalidad de controlar la ICC: movimiento de la mano izquierda y mano derecha; movimiento de la mano izquierda y de la lengua; y movimiento de la mano izquierda y pie. Tales combinaciones de movimientos producen patrones reconocibles y diferenciables en la actividad eléctrica cerebral que, después de su detección, son mapeados de una forma no-transparente. Por ejemplo, en ${ }^{[46]}$, la comparación entre la activación/desactivación correspondiente al movimiento de la mano izquierda y lengua se empleó para seleccionar una letra del alfabeto. Esta incongruencia entre tarea mental y comando de control podría ocasionar una alteración en el sentido de autonomía que afectaría el desempeño del usuario al interactuar con la ICC. La congruencia se logra, entonces, cuando el ambiente de una ICC se modifica como si los movimientos imaginarios estuvieran siendo realmente ejecutados [47]. Ejemplo de ello es el estudio de [48], donde el movimiento imaginado por los participantes (movimiento del brazo derecho) correspondió con la acción ejecutada por el sistema (movimiento de un brazo en pantalla y estimulación háptica del usuario) al detectar el estado de imaginación. A pesar de que el objetivo de los investigadores no fue comparar la calidad de la comunicación entre una tarea congruente en 


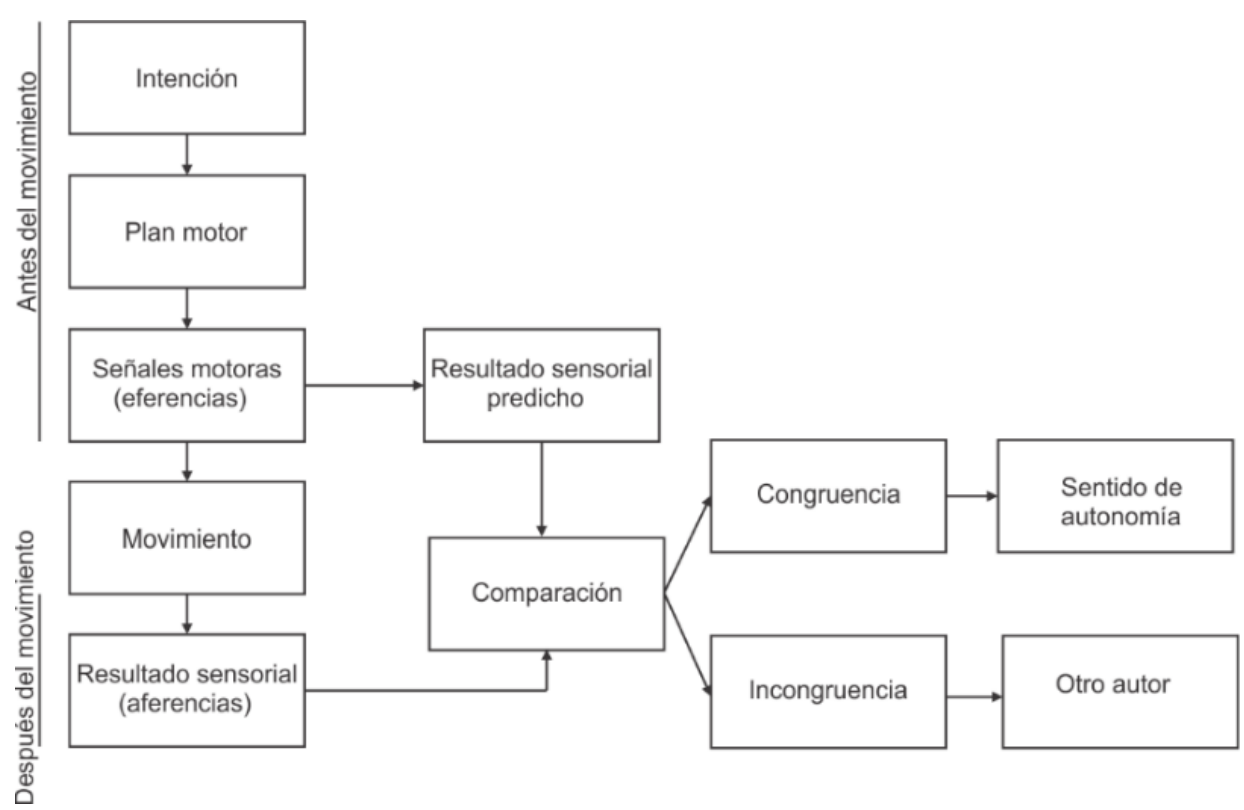

FIGURA 3. Teoría de monitoreo central. Esta teoría propone que la incongruencia entre el resultado sensorial predicho antes de realizar un movimiento y el resultado sensorial posterior a la ejecución del movimiento es la responsable de que un individuo atribuya el movimiento a un agente distinto a él. Imagen modificada de ${ }^{[51]}$.

comparación con una incongruente, el desempeño del sistema sugiere que la congruencia debe ser un factor a considerar en los posteriores rediseños de las tareas de control para ICCs.

\section{Sentido de Autonomía}

El sentido de autonomía es el sentido de ser el autor de una acción. Por ejemplo, el sentido de ser el responsable de que algo se mueva, o de generar un cierto pensamiento ${ }^{[49]}$. Involucra un fuerte componente eferente porque los comandos motores preceden al movimiento voluntario ${ }^{[50]}$. Una de las teorías más populares para explicarlo es la "teoría de monitoreo central" (ver Figura 3). De acuerdo con esta, dos tipos de modelos internos son implementados por el sistema motor central; los llamados modelos directo e inverso. El sentido de autonomía se basa particularmente en el primero, el cual usa una copia de los comandos motores para pronosticar las respectivas consecuencias sensoriales. Según este modelo, la congruencia del resultado predicho con el real produciría la atribución de la acción a uno mismo. Mientras que la incongruencia indicaría que otro agente ha sido el causante de la acción ${ }^{[51]}$. No obstante, es necesario indicar que también existe evidencia de que no depende únicamente del resultado de una acción, sino que podría ser desencadenado antes de que la acción se realice [52] [53].

En ${ }^{[54]}$, los participantes del grupo experimental que realizaron una tarea de entrenamiento diseñada para producir, entre otras cosas, un alto sentido de autonomía se desempeñaron significativamente mejor en la tarea de evaluación en línea y también obtuvieron una mayor precisión en la clasificación que el grupo control entrenado con un protocolo estándar. Por lo que, se propone que al controlar un dispositivo externo, como una ICC, el papel que juega el sentido de autonomía es de gran relevancia debido a que influye en el interés de las personas hacia la tecnología y, por lo tanto, en su compromiso con la tarea y desempeño ${ }^{[44]}$. En ${ }^{[44]} y^{[55]}$, se considera que los protocolos de entrenamiento deben ser capaces de provocar un alto sentido de auto- 
nomía en los participantes para que alcancen un buen desempeño, puesto que reduce la sensación de ansiedad producida por el sistema y esto permite que los participantes destinen más recursos a la tarea en lugar de preocuparse por su desempeño.

Esto cobra particular importancia en la actualidad, donde la investigación y uso cada vez más frecuente de ICC implantables, determina que estas logren un control más sofisticado de los circuitos neurales subyacentes, pero su "ensamble funcional" con el sentido de control de sí mismo en el paciente, podría afectarlo psicológicamente si no resulta apropiado ${ }^{[56]}$. Algunos autores han interpretado la percepción del sentido de autonomía y sus efectos psicológicos en pacientes usuarios de ICC implantables dentro de un continuum que transcurre desde una alta confianza y sentido de autocontrol, hasta un significativo estado de angustia y de percepción de daño causado por la ICC [57].

\section{Retroalimentación Multisensorial}

Como podrá comprenderse de los conceptos antes expresados respecto al sentido de autonomía, éste refleja un sentimiento multifactorial y depende, en una gran medida, del intervalo temporal que transcurre entre la acción y su efecto constatable. Si no existe una sincronización temporal entre ambos, entonces la predictibilidad de la consecuencia de la acción disminuirá y, por ende, el sentido de autocontrol sobre la misma. Por eso es de vital importancia que el individuo reciba retroalimentación respecto a su decisión y consecuencias.

Para interactuar de manera exitosa con el medio ambiente, el cerebro integra múltiples pistas sensoriales para formar una representación coherente del mundo ${ }^{[58]}$. Por lo que, durante la ejecución de una tarea es deseable presentar información al participante sobre su desempeño (retroalimentación), con el propósito de ayudarle a modular su actividad cerebral en los intentos posteriores. En estudios como los de [59], la retroalimentación háptica y visual, en conjunto, ha mostrado ser útil para incrementar el desempeño del usuario al usar la ICC. Lo mismo encontraron los estudios de ${ }^{[60]}$, que demuestran que una integración multisensorial coherente puede mejorar el desempeño del sistema. En dicho estudio, a los participantes se les presentó retroalimentación audiovisual mientras controlaban la caminata de un robot. La condición que menos tiempo requirió para dirigir el robot hacia un objetivo fue aquella donde se les presentó información audiovisual coherente. Es decir, la condición en la cual los participantes escuchaban los pasos del robot al mismo tiempo que veían al robot desplazarse. Un ejemplo más de ello, lo constituye el estudio conducido por ${ }^{[61]}$, quienes les pidieron a un grupo de sujetos que indicaran si el sonido de los pasos que sentían era producido por ellos o no. En el caso en que los pasos se sincronizaron con el sonido de los mismos, aseguraron que eran ellos los que lo producían (alto sentido de autonomía), pero no así cuando los estímulos no estaban sincronizados. Finalmente, en ${ }^{[48]}$, se usaron estímulos hápticos que provocaron la ilusión de movimiento de los miembros superiores y estímulos visuales fueron empleados como forma de retroalimentación en una ICC basada en IM. Los autores de este trabajo mostraron que la retroalimentación multisensorial congruente aumentó significativamente el desempeño del sistema ICC con respecto a la condición de retroalimentación unisensorial (visual).

En este contexto, no es extraño entonces que en los últimos años haya crecido significativamente el uso de la retroalimentación acoplada a la operación de las ICC y proporcionada a través de distintos sistemas sensoriales o una combinación de estos (e.g. visual: [62], [63]; auditiva: ${ }^{[64]}$; propioceptiva: ${ }^{[65]}$; vibrotáctil y visual: ${ }^{[48]}$; visual y auditiva: ${ }^{[60]}$. En ${ }^{[66]}$, se recomienda que la información entregada a través de los distintos canales sensoriales como retroalimentación debe poder ser interpretada de la misma manera, usando el mismo tipo de estrategia mental para que sea efectiva. 
Por tanto, se sugiere que la información presentada como retroalimentación debe: poder ser interpretada de la misma manera, la misma cantidad de contenido explicativo y debe ser redundante para que el usuario pueda relacionarlas ${ }^{[66][67]}$.

\section{Ambientes Ecológicos}

Se considera que el control de una ICC es una habilidad que debe ser aprendida y dominada por el mismo usuario ${ }^{[41]}$. Éste debe producir patrones de actividad cerebral que puedan ser identificados por algún método de clasificación. Para ello, se le pide, en una sesión de entrenamiento, que ejecute cierta IM durante períodos determinados de tiempo. De esta manera, es posible relacionar el registro de EEG con el momento de la ejecución de la IM y, después, alimentar a un algoritmo de aprendizaje automático para la clasificación en línea. Sin embargo, la ausencia de un mapeo transparente y de una retroalimentación multisensorial congruente durante la sesión de entrenamiento podría impedir que la habilidad de controlar una ICC sea dominada por el sujeto, afectando el desempeño del sistema. Con base en lo anterior, se ha propuesto que las tareas de las sesiones de entrenamiento deberían incluir condiciones semejantes a las del mundo real. Es decir, incluir un ambiente ecológico. Ejemplo de ello es el trabajo de ${ }^{[54]}$, en el cual el entrenamiento fue transferido a un ambiente inmersivo de realidad virtual. Esta última permite tener una retroalimentación más natural, en este caso un cuerpo humano. Si los participantes pueden creer que la representación corporal que se les presenta es suya, la retroalimentación podría ser llevada a cabo de una manera más natural y efectiva. Así lo indican los resultados de su trabajo, donde se observa un efecto positivo del ambiente de entrenamiento.

Un ambiente ecológico se lograría, entonces, cuando la IM esté basada en un movimiento automatizado por el participante, donde dicho movimiento sea mapeado de forma transparente, se provea de retroalimentación multisensorial correlacionada al movimiento y la tarea de entrenamiento se diseñe con un propósito claro para el usuario.

\section{IMPACTO SOCIAL}

La importancia de hacer realidad la tecnología de asistencia basada en sistemas ICC, radica en el impacto que tendría sobre la población mexicana que presenta algún tipo de discapacidad. De acuerdo a las estadísticas del INEGI (Tabla 1), 7.2 millones de mexicanos tienen dificultad para llevar a cabo actividades básicas, mientras que $\mathbf{1 5 . 9}$ millones tienen alguna limitación moderada, representando el 6\% del total de la población. Si los sistemas ICC llegaran a ser una realidad accesible, no solo las personas con dificultad de movimiento (hasta el 80\% de la población con discapacidad mayor a 60 años) serían beneficiadas, sino que de acuerdo a las aplicaciones de los sistemas ICC propuestas a la fecha, se podrían dar alternativas a personas con dificultades comunicativas (hasta $46 \%$ de la población con discapacidad entre 0 y 14 años), de aprendizaje (hasta $45 \%$ de la población con discapacidad mayor a 60 años), y mentales (hasta $28 \%$ de la población con discapacidad entre 15 y 29 años). Todavía más, existe la posibilidad de mejorar la calidad de vida de personas que a la fecha carecen de alternativa alguna, es decir, todas aquellas personas con limitaciones o dificultades para atenderse a sí mismos (hasta $37 \%$ de la población con discapacidad entre 0 y 14 años).

\section{Efectividad y adaptabilidad en dispositivos de asistencia motora}

De acuerdo a las estadísticas de la Tabla 1, la discapacidad motora (tanto superior como inferior) suma el porcentaje más alto del total de la población con capacidades diferentes. Dicha discapacidad además de incrementarse con la edad, también se relaciona directamente con accidentes (véase Tabla 2). Entre los dispositivos de asistencia motora se encuentran las órtesis, que son sistemas de fuerza, diseñados para contro- 
TABLA 1. Porcentaje de población con discapacidad, de acuerdo a la edad y el tipo de discapacidad

\begin{tabular}{lcccc}
\hline \multirow{2}{*}{ Tipos de discapacidad } & \multicolumn{2}{c}{ Grupos de edad } \\
& $\mathbf{0}$ a $\mathbf{1 4}$ años & $\mathbf{1 5}$ a 29 años & $\mathbf{3 0}$ a 59 años & $\mathbf{6 0}$ años y más \\
\hline Caminar, subir o bajar usando sus piernas & 36.2 & 32.1 & 56.2 & 81.3 \\
\hline Ver (aunque usen lentes) & 26.9 & 44.6 & 58.2 & 67.2 \\
\hline Mover o usar sus brazos y manos & 14.1 & 18.2 & 28.5 & 42.7 \\
\hline Aprender, recordar o concentrarse & 40.8 & 31.5 & 32.1 & 44.6 \\
\hline Escuchar (aunque usen aparato auditivo) & 13.4 & 18.5 & 24.2 & 46.9 \\
\hline Bañarse, vestirse o comer & 37.4 & 16.4 & 14.5 & 29.3 \\
\hline Hablar o comunicarse & 45.6 & 28.5 & 13.4 & 14.0 \\
\hline Problemas emocionales o mentales & 26.6 & 28.0 & 20.1 & 16.3 \\
\hline
\end{tabular}

Nota: Una persona puede tener más de una discapacidad, por lo que cada grupo supera el $100 \%$. Fuente: INEGI. Encuesta Nacional de la Dinámica Demográfica 2014. Base de Datos.

lar, corregir o compensar una deformidad ósea, deformidad de esfuerzo, o ausencia de fuerza en el cuerpo. Existen otros dispositivos de asistencia tales como sillas de ruedas, caminadoras, bastones, etc. También existen prótesis para personas con una disparidad por pérdida de algún miembro o por algún defecto congénito. Actualmente, varios de estos dispositivos cuentan con implementaciones robóticas o de inteligencia artificial que han incrementado su efectividad y adaptabilidad. Sin embargo, muchas de estas implementaciones se basan en el uso de la actividad eléctrica muscular, la cual solo puede ser registrada en pacientes que no tienen un daño en el sistema nervioso periférico. Para todos aquellos pacientes que solo cuentan con la actividad eléctrica de su SNC (ej., cuadriparesia, cuadriplejia), los sistemas ICC son la única alternativa de llegar a tener un dispositivo de asistencia motora eficiente y adaptable.

\section{Rehabilitación}

En los últimos años, la investigación en ICC también ha ganado interés en el campo de la rehabilitación, debido a la naturaleza misma del sistema. Es decir, el funcionamiento de una ICC depende de la modulación de la actividad neuronal, lo cual conlleva cambios de neuroplasticidad que pudiesen ser aprovechados en el área de rehabilitación. El uso de una ICC como medio de rehabilitación no ha sido completamente validado aún; sin embargo, existen evidencias científicas que dan soporte a esta hipótesis como en el caso de ${ }^{[68]}$, donde fue demostrado que la estimulación eléctrica periférica sincronizada con los MRCP incrementa la excitabilidad cortico-espinal, promoviendo, entonces, una plasticidad neural específica que ya había sido postulada con anterioridad [69] [70]. Se ha demostrado que una neurorehabilitación basada en sistemas ICC explora las propiedades neuroplásticas del cerebro a través de tareas imaginarias motoras. Esto quiere decir que el uso de una ICC como medio de rehabilitación puede dar paso a una mejora en los procesos de aprendizaje y elaboración de funciones motoras ${ }^{[16]}$. También se ha sugerido que la imaginación de movimientos específicos podría ser una herramienta de apoyo para la rehabilitación de pacientes que presentan un dolor físico intenso ${ }^{[71] ~[72] . ~ M u c h o s ~ c i e n t i ́ f i c o s ~ i n v o l u c r a d o s ~}$ en la investigación de la rehabilitación pasiva (terapias 
TABLA 2. Enfermedades y traumatismos que afectan al sistema músculo-esquelético por tipo de padecimiento y frecuencia

\begin{tabular}{lccc}
\hline Grupos & Frecuencia & \% Parcial & \% Global \\
\hline Facturas & 2374 & 20.0 & 10.93 \\
Artropatías & 2287 & 19.3 & 10.53 \\
Trastornos de los tejidos blandos & 2271 & 19.2 & 10.46 \\
Dorsopatías & 2121 & 17.9 & 9.77 \\
Enfermedades del sistema nervioso & 690 & 5.8 & 3.18 \\
Osteopatías y condropatías & 675 & 5.7 & 3.11 \\
Malformaciones congénitas del sistema osteomuscular & 620 & 5.2 & 2.86 \\
Deformidades musculo esqueléticas (adquiridas) & 329 & 2.8 & 1.52 \\
Tumores del sistema musculo esquelético & 312 & 2.6 & 1.44 \\
Amputaciones & 166 & 1.4 & 0.76 \\
\hline Total & $\mathbf{1 1 8 4 5}$ & $\mathbf{1 0 0 . 0}$ & $\mathbf{5 4 . 5 6}$ \\
\hline
\end{tabular}

basadas en imaginación motora) han especificado que éste tipo de rehabilitación no sólo involucra al área de consulta hospitalaria, sino que también depende de herramientas suplementarias como audios y/o estímulos visuales que refuercen el proceso imaginario motriz, además de instructivos específicos que guíen al paciente para realizar dicha rehabilitación desde su hogar [73] [74] [75].

En resumen, un sistema ICC puede llegar a utilizarse para controlar un dispositivo auxiliar, para reemplazar una función motora perdida, para manipular miembros robóticos o para dar paso a la neuroplasticidad que conlleve a la recuperación motora (efecto neuromodulador). Todas estas aplicaciones beneficiarían a personas que tienen una discapacidad motora (Tabla 1) o que padecen alguna enfermedad, o algún traumatismo, que afecte a su sistema músculo-esquelético (Tabla 2).

\section{CONCLUSION}

La convergencia entre el desarrollo incesante de nuevas y más potentes herramientas de cálculo y procesamiento matemático, junto al avance en el conocimiento y la comprensión de las bases neurales de la cognición, la actividad motora y el modo en que estas se expresan funcionalmente, han permitido la generación de intermediarios computacionales (ICC) que hoy constituyen la mejor y más lograda esperanza de restaurar la función y calidad de vida a pacientes con una amplia variedad de lesiones y limitaciones neurológicas, entre otras muchas importantes aplicaciones.

El futuro de estas ICC y la eficiencia con que logren el objetivo para el que fueron diseñadas, parece depender más que nunca de factores relacionados a la percepción subjetiva del usuario, su adaptación al manejo de las ICC y el proceso de internalizarlas como propias en su espacio personal y su psique. 


\section{REFERENCIAS}

[1] N. Alamdari, A. Haider, R. Arefin, A. K. Verma, K. Tarakolian y R. Fazel-Rezai, «A review of methods and applications of brain computer interface systems,» de IEEE International Conference on Electro Information Technology (EIT), Dakota, 2016.

[2] L. M. Alonso-Valerdi, R. A. Salido-Ruiz y R. A. Ramirez-Mendoza, «Motor imagery based brain-computer interfaces: an emerging technology to rehabilitate motor deficits,» Neuropsychologia, vol. 79, pp. 354-363, 2015.

[3] D. Lulé, Q. Noirhomme, S. C. Kleih, C. Chatelle, S. Halder, A. Demertzi, M. Bruno, O. Gosseries, A. Vanhaudenhuyse, C. Schnakers, M. Thonnard, A. Soddu, A. Kübler y S. Laureys, «Probing command following in patients with disorders of consciousness using a brain-computer interface,» Clinical Neurophysiology, vol. 124, no 1, pp. 101-106, 2013.

[4] C. G. Lim, T. S. Lee, C. Guan, D. S. S. Fung, Y. Zhao, S. S. W. Teng, H. Zhang y K. R. R. Krishman, «A brain-computer interface based attention training program for treating attention deficit hyperactivity disorde,» PLoS ONE, vol. 7, no 10, p. e46692, 2012.

[5] T. P. Cothran y J. E. Larson, «Brain-computer interface technology for schizophrenia,» Diagnosis, vol. 8, no 4, pp. 337-340, 2012.

[6] E. V. C. Friedrich, N. Suttie, A. Sivanathan, T. Lim, S. Louchart y J. A. Pineda, «Brain-computer interface game applications for combined neurofeedback and biofeedback treatment for children on the autism spectrum,» Frontiers in Neuroengineering, vol. 7, n 21, 2014.

[7] F. Lotte, C. S. Nam y A. Nijholt, «Introduction: Evolution of BrainComputer Interfaces,» de Brain-Computer Interfaces Handbook: Technological and Theoretical Advances, Boca Raton, Taylor \& Francis, 2018, pp. 1-8.

[8] R. P. N. Rao, «Introduction,» de Brain-Computer Interfacing, New York, Cambridge University Press, 2013, pp. 1-3.

[9] C. Jeunet, E. Jahanpour y F. Lotte, «Why standard brain-computer interface (BCI) training protocols should be changed: an experimental study," Journal of Neural Engineering, vol. 13, $\mathrm{n}^{\mathrm{O}} 3$, p. $036024,2016$.

[10] S. F.-R. R. \&. A. V. Amiri, «A review of hybrid brain-computer interface systems.,» Advances in Human-Computer Interaction, pp. 1-8, 2013.

[11] C. Jeunet, A. Cellard, S. Subramanian, M. Hachet, B. N'Kaoua y F. Lotte, «How Well Can We Learn With Standard BCI Training Approaches? A Pilot Study,» de 6th International Brain-Computer Interface Conference, Graz, 2014.

[12] F. Lotte, F. Larrue y C. Mühl, «Flaws in current human training protocols for spontaneous brain-computer interfaces: lessons learned from instructional design,» Frontiers in Human Neurosciences, vol. 7, nº 568, pp. 9-19, 2013.

[13] C. Jeunet y F. Lotte, «Why and How to Use Intelligent Tutoring Systems to Adapt MI-BCI Training to Each User,» de 6th International BCI Meeting, Graz, 2016.

[14] F. Lotte y C. Jeunet, «Towards improved BCI based on human learning principles,» de 3rd International Winter Conference on Brain-Computer Interfaces, Korea, 2015.

[15] A. S. M. F. T. Y. Y. C. \&. G. L. Battison, «Effectiveness of Subject Specific Instruction on Mu-based Brain-Computer Interface Training,» de NEBEC, 2015.
[16] J. Wolpaw y E. W. Wolpaw, «Brain-computer interfaces: principles and practice,» OUP , USA, 2012.

[17] T. O. Zander, C. Kothe, S. Jatzev y M. Gaertner, «Enhancing humancomputer interaction with input from active and passive braincomputer interfaces,» Brain-Computer Interfaces, vol. Springer, no London, pp. 181-199, 2010.

[18] C. M. Bishop, Patter Recognition and Machine Learning, Cambridge: Springer, 2006.

[19] C. A. Porro, M. P. Francescato, V. Cettolo, M. E. Diamond, P. Baraldi, C. Zuiani, M. Bazzocchi y P. E. di Prampero, «Primary Motor and Sensory Cortex Activation during Motor Imagery: A Functional Magnetic Resonance Imaging Study,» The Journal of Neuroscience, pp. 7688-7698, 1996.

[20] M. Jeannerod, «The representing brain: Neural correlates of motor intention and imagery,» Behavioral and Brain sciences , pp. 187202, 1994.

[21] D. J. McFarland, L. A. Miner, T. M. Vaughan y J. R. Wolpaw, «Mu and beta rhythm topographies during motor imagery and actual movements,» Brain topography, pp. 177-186, 2000.

[22] G. Pfurtscheller y N. Christa, «Motor imagery and direct braincomputer communication,» Proceedings of the IEEE , pp. 11231134, 2001.

[23] S. Lemn, S. Christin y C. Gabriel, «Lemm, Steven, Christin Schafer, and Gabriel Curio. "BCI competition 2003-data set III: probabilistic modeling of sensorimotor/spl mu/rhythms for classification of imaginary hand movements,» IEEE Transactions on Biomedical Engineering, pp. 1077-1080, 2004.

[24] G. Pfurtscheller, C. Brunner, A. Schlögl y F. L. Da Silva, «Pfurtscheller, Gert, et al. "Mu rhythm (de) synchronization and EEG single-trial classification of different motor imagery tasks,» NeuroImage, pp. 153-159, 2006.

[25] Y. Wang y P. M. William, «Wang, Youde, and William P. Morgan. "The effect of imagery perspectives on the psychophysiological responses to imagined exercise,» Behavioural brain research, pp. 167-172, 1992.

[26] H. H. Ehrsson, G. Stefan y N. Eiichi, «Ehrsson, H. Henrik, Stefan Geyer, and Eiichi Naito. "Imagery of voluntary movement of fingers, toes, and tongue activates corresponding body-partspecific motor representations,» Journal of neurophysiology, pp. 3304-3316, 2003.

[27] J. Decety, «Decety, Jean. «Do imagined and executed actions share the same neural substrate?,» Cognitive brain research, pp. 87-93, 1996.

[28] M. Jeannerod, «Mental imagery in the motor context,» Neuropsychologia, pp. 1419-1432, 1995.

[29] M. Jeannerod y J. Decety, «Mental motor imagery: a window into the representational stages of action,» Current opinion in neurobiology, pp. 727-732, 1995.

[30] A. M. L. K. F. \&. K. E. A. Seeland, «Spatio-Temporal Comparison Between ERD/ERS and MRCP-based Movement Prediction,» BIOSIGNALS-15, pp. 219-226, 2015.

[31] G. S. F. L. d. \&. L. d. S. F. H. Pfurtscheller, «Event-related EEG/MEG synchronization and desynchronization: basic principles,» Clinical Neurophysiology, pp. 1842-1857, 1999. 
[32] C. S. e. a. Nam, «Movement imagery-related lateralization of eventrelated (de)synchronization (ERD/ERS): Motor imagery duration effects.,» Clinical Neurophysiology, pp. 567-577, 2011.

[33] V. G. L. A. \&. Y. Y. C. Corbit, «Improving Mu Rhythm BrainComputer Interface Performance by Providing Specific Instructions for Control.,» de NEBEC 2013, 2013.

[34] H. \&. H. M. Shibasaki, «What is the Bereitschaftspotential?,» Clinical Neurophysiology, pp. 2341-2356, 2006.

[35] R. I. R. B. J. \&. P. J. G. Cunnington, «Movement-related potentials associated with movement preparation and motor imagery,» Experimental Brain Research, pp. 429-436, 1996.

[36] K. G. Y. N. O. F. \&. F. D. Dremstrup, «Movement-related cortical potentials and their application in brain-computer interfacing,» Wiley-IEEE, pp. 253-266, 2013.

[37] I. N. \&. G. D. Angulo-Sherman, «Effect of different feedback modalities in the performance of brain-computer interfaces,» de CONIELECOMP 2014, 2014.

[38] J. J. C. \&. L. F. Schumacher, «Towards Explanatory Feedback for User Training in Brain-Computer Interfaces,» Hong Kong, p. s.n, 2015.

[39] T. H. E. M. \&. K. A. Kaufmann, «Comparison of tactile, auditory, and visual modalitu for brain-computer interface use: a case study with a patient in the locked-in state,» Frontiers in Neuroscience, p. s.n., 2013.

[40] F. \&. J. C. Lotte, «Towards improved BCI based on human learning principles,» de Brain-Computer Interface (BCI), 3rd International Winter Conference on,, 2015.

[41] J. R. Wolpaw y E. W. Wolpaw, Brain-Computer Interfaces: Something New Under the Sun, New York: Oxford University Press, 2012.

[42] F. Lotte y C. Jeunet, «Lotte, Fabien, and Camille Jeunet. "Towards improved BCI based on human learning principles,» de The 3rd International Winter Conference on Brain-Computer Interface, 2015.

[43] C. Jeunet, B. N'Kaoua, R. N’Kambou y F. Lotte, «Why and How to Use Intelligent Tutoring Systems to Adapt MI-BCI Training to Each User,» de 6th International BCI Meeting, Asilomar, United States, 2016.

[44] R. Vlek, J. P. van Acken, E. Beursken, L. Roijendijk y P. Hselager, «BCI and a User's Judgment of Agency,» de Brain-ComputerInterfaces in their ethical, social and cultural contexts, Dordrecht, Springer, 2014, pp. 193-202.

[45] M. Quek, J. Höhne, R. Murray-Smith y M. Tangermann, «Quek, Melissa, et al. "Designing future BCIs: Beyond the bit rate,» de Towards Practical Brain-Computer Interfaces, Berlin, Springer, 2012, pp. 173-196.

[46] C. Guger, G. Edlinger, W. Harkam, I. Niedermayer y G. Pfurtscheller, «How many people are able to operate an EEG-based brain-computer interface (BCI)?,» IEEE transactions on neural systems and rehabilitation engineering, pp. 145-147, 2003.

[47] L. M. Alonso-Valerdi y A. A. González-Garrido, «Characterizing Motor System to Improve Training Protocols Used in BrainMachine Interfaces Based on Motor Imagery,» de Cognitive and Computational Neuroscience: Principles, Algorithms and Applications, London, IntechOpen, 2018, pp. 57-76.
[48] M. Barsotti, D. Leonardis, N. Vanello, M. Bergamasco y A. Frisoli, «Effects of continuous kinaesthetic feedback based on tendon vibration on motor imagery BCI performance,» IEEE Transactions on Neural Systems and Rehabilitation Engineering, vol. 26, no 1, pp. 105-114, 2017.

[49] S. Gallagher, «Philosophical conceptions of the self: implications for cognitive science,» Trends in cognitive sciences, pp. 14-21, 2000.

[50] M. Tsakiris, P. Gita y H. Patrick, «Tsakiris, Manos, Gita Prabhu, and Patrick Haggard. "Having a body versus moving your body: How agency structures body-ownership,» Consciousness and cognition, pp. 423-432, 2006.

[51] N. David, N. Albert y V. Kai, «The "sense of agency" and its underlying cognitive and neural mechanisms,» Consciousness and cognition, pp. 523-534, 2008.

[52] S. Gallagher, «Multiple aspects in the sense of agency,» New ideas in psychology, pp. 15-31, 2012.

[53] M. Synofzik, V. Gottfried y N. Albert, «Beyond the comparator model: a multifactorial two-step account of agency,» Consciousness and cognition, pp. 219-239, 2008.

[54] F. Škola y F. Liarokapis, «Embodied VR environment facilitates motor imagery brain-computer interface training,» Computers \& Graphics, vol. 75, pp. 59-71, 2018.

[55] C. Jeunet, B. N'Kaoua y F. Lotte, «Towards a cognitive model of MI-BCI user training,» de International Graz BCI Conference „, 2017.

[56] W. Glannon y C. Ineichen, «Philosophical aspects of closed-loop neuroscience,» InClosed loop neuroscience, vol. 1, no 1, pp. 259$270,2016$.

[57] F. Gilbert, M. Cook, T. O'Brien y J. Illes, «Embodiment and estrangement: results from a first-in-human "intelligent BCI" trial,» Science and Engineering Ethics, vol. 25, no 1, pp. 83-96, 2019.

[58] E. Tidoni, P. Gergondet, A. Kheddar y S. M. Aglioti, «"Audio-visual feedback improves the BCI performance in the navigational control of a humanoid robot,» Frontiers in neurorobotics, p. 20, 2014.

[59] M. Gomez-Rodriguez, J. Peters, J. Hill, B. Schölkopf, A. Gharabaghi y M. Grosse-Wentrup, "Closing the sensorimotor loop: haptic feedback facilitates decoding of motor imagery,» Journal of neural engineering, p. 036005, 2011.

[60] E. Tidoni, P. Gergondet, G. Fusco, A. Kheddar y S. Aglioti, «The role of audio-visual feedback in a thought-based control of a humanoid robot: a BCI study in healthy and spinal cord injured people,» Neural Systems and Rehabilitation Engineering, pp. 772-781, 2016.

[61] F. Menzer, A. Brooks, P. Halje, C. Faller, M. Vetterli y O. Blanke, «Feeling in control of your footsteps: conscious gait monitoring and the auditory consequences of footsteps,» Cognitive neuroscience, vol. 1, no 3, pp. 184-192, 2010.

[62] E. E. Fetz, «Operant conditioning of cortical unit activity,» Science, vol. 163, no 3870, pp. 955-958, 1969.

[63] M. C. Dadarlat, J. E. O'doherty y P. N. Sabes, «A learning-based approach to artificial sensory feedback leads to optimal integration,» Nature Neuroscience, vol. 18, nº 1, p. 138, 2015.

[64] R. J. Schafer y T. Moore, «Selective attention from voluntary control of neurons in prefrontal cortex,» Science, vol. 332, n⿳0 6037, pp. 1568-1571, 2011. 
[65] A. J. Suminski, D. C. Tkach, A. H. Fagg y N. G. Hatsopoulos, «Incorporating feedback from multiple sensory modalities enhances brain-machine interface control,» Journal of Neuroscience, vol. 30, no 50, pp. 16777-16787, 2010.

[66] S. Ainsworth, «DeFT: A conceptual framework for considering learning with multiple representations,» Learning and instruction, vol. 16, no 3, pp. 183-198, 2006.

[67] F. Lotte, F. Larrue y C. Mühl, «Flaws in current human training protocols for spontaneous brain-computer interfaces: lessons learned from instructional design,» Frontiers in human neuroscience, vol. 7, p. 568, 2013.

[68] M. Jochumsen, M. S. Navid, R. W. Nedergaard, N. Signal, U. Rashid, A. Hassan, H. Haavik, D. Taylor y I. K. Niazi, «Self-Paced Online vs. Cue-Based Offline Brain-Computer Interfaces for Inducing Neural Plasticity,» Brain Sciences, vol. 9, no 6, p. 127, 2019.

[69] N. Mrachacz-Kersting y S. Aliakbaryhosseinabadi, «Comparison of the efficacy of a Real-time and Offline Associative BrainComputer-Interface,» Frontiers in Neuroscience, vol. 12, no 1, p. 455, 2018.

[70] N. Mrachacz-Kersting, S. R. Kristensen, I. K. Niazi y D. Farina, «Precise temporal association between cortical potentials evoked by motor imagination and afference induces cortical plasticity,» The Journal of Physiology, vol. 590, nº 7, pp. 1669-1682, 2012.

[71] S. J. Snodgrass, N. R. Heneghan, H. Tsao, P. T. Stanwell, D. A. Rivett y P. M. Van Vliet, «Recognising neuroplasticity in musculoskeletal rehabilitation: A basis for greater collaboration between musculoskeletal and neurological physiotherapists,» Manual Therapy, vol. 19, nº 6, pp. 614-617, 2014.

[72] J. Harris y A. Hebert, «Utilization of motor imagery in upper limb rehabilitation: a systematic scoping review,» Clinical Rehabilitation, vol. 29, no 11, pp. 1092-1107, 2015.

[73] C. Zimmermann-Schlatter, C. Schuster, M. A. Puhan, E. Siekierka y J. Steurer, «Efficacy of motor imagery in post-stroke rehabilitation: a systematic review,» Journal of Neuroengineering and Rehabilitation, vol. 5, no 1, p. 8, 2008.
[74] M. Letswaart, M. Johnston, H. C. Dijkerman, S. Joice, C. L. Scott, R. S. McWalter y S. Hamilton, «Mental practice with motor imagery in stroke recovery: randomized controlled trial of efficacy,» Brain, vol. 134, nº 5, pp. 1373-1386, 2011.

[75] D. Caligiore, M. Mustile, G. Spalletta y G. Baldassarre, «Action observation and motor imagery for rehabilitation in Parkinson's disease: A systematic review and an integrative hypothesis,» Neuroscience \& Biobehavioral Reviews, vol. 72, pp. 210-222, 2016.

[76] H. W. R. B. G. \&. B. A. Bashashati, «Comparing Different Classifiers in Sensory Motor Brain Computer Interfaces,» PLoS ONE, 2015.

[77] I. J. N. J. M. F. J. D. K. \&. F. D. Khan, «Detection of MovementRelated Cortical Potencials Based on Subject-Independent Training,» Med Biol Eng Compu, pp. 507-512, 2013.

[78] L. W. I. M. K.-R. \&. S. W. Frølich, «Investigating effects of different artefact types on Motor Imagery BCI.,» de EMBC Conference, 2015.

[79] T. Hinterberger, S. Schmidt, N. Neumann, J. Mellinger, B. Blankertz, G. Curio y N. Birbaumer, «Brain-computer communication and slow cortical potentials,» IEEE Transactions on Biomedical Engineering, pp. 1011-1018, 2004.

[80] T. Hinterberger, N. Neumann, M. Pham, A. Kübler, A. Grether, N. Hofmayer, B. Wilhelm, H. Flor y N. Birbaumer, «A multimodal brain-based feedback and communication system,» Experimental Brain Research, pp. 521-526, 2003.

[81] M. Pham, T. Hinterberger, N. Neumann, A. Kübler, N. Hofmayer, B. Wilhelm, J. Vatine y N. Birbaumer, «An Auditory Brain-Computer Interface Based on the Self-Regulation of Slow Cortical Potentials,» Neurorehabilitation and Neural Repair, pp. 206-218, 2005.

[82] B. Z. Allison, C. Brunner, C. Altstätter, I. C. Wagner, S. Grissmann y C. Neuper, «A hybrid ERD/SSVEP BCI for continuous simultaneous two dimensional cursor control,» Journal of neuroscience methods, pp. 299-307, 2012. 